\title{
The Late Quaternary \\ vegetational and environmental history \\ of western tropical Africa: \\ the eastern Benin Basin, Lagos, Nigeria
}

\author{
Peter Adegbenga Adeonipekun ${ }^{1}$, Tiwalade Adeyemi Adeniyi ${ }^{1}$, Jacob Mateawo ${ }^{2}$, \\ Biola Agbalaya ${ }^{1}$ \\ ${ }^{1}$ University of Lagos, Department of Botany; Nigeria; e-mail: p1adeonipekun@yahoo.com, tiwaladeadeniyi@gmail.com \\ ${ }^{2}$ GEC Energy Solutions Limited; Lagos, Nigeria
}

(C) 2017 Authors. This is an open access publication, which can be used, distributed and reproduced in any medium according to the Creative Commons CC-BY 4.0 License requiring that the original work has been properly cited.

Received: 26 March 2017; accepted: 19 September 2017

\begin{abstract}
A palynological study of a $30 \mathrm{~m}$ borehole within the Akoka campus of the University of Lagos, Akoka, Nigeria revealed Late Quaternary paleo-vegetational, climatic and environmental changes. Sixteen samples at a regular interval of $2 \mathrm{~m}$ were subjected to standard palynological and sedimentological techniques. Six pollen zones representing six paleoclimatic phases within the Late Quaternary period were recognized. The age model is based on two radiocarbon dates $\left(6 \mathrm{~m}: 5,495 \pm 25{ }^{14} \mathrm{C}\right.$ yr and $\left.12 \mathrm{~m}: 15,660 \pm 40{ }^{14} \mathrm{C} \mathrm{yr}\right)$ and, by extrapolation, the sequence spans approximately the last 35,000 yr BP. The African maximum transgression and the African Humid Period (wet paleoclimate maximum) - the Nouaktchottian, and Pre-Nouaktchottian respectively - were recognized, while the Ogolian II (Erg II) was stratigraphically recognized and the Upper Inchirian extrapolated. Recovered diatom frustules with other proxies were employed for paleoclimatic and paleoecological deductions. Four hydrological cycles and three paleoecological settings were identified. Results from this work indicate the co-eval record of paleoclimatic events across Africa, suspected cooling during the African maximum transgression in Nigeria and the usefulness of coastal freshwater swamp sediments for paleoclimate studies.
\end{abstract}

Keywords: paleoclimate, paleoecology, paleovegetation, palynology, sedimentology, Lagos Coastal Area, Late Quaternary

\section{INTRODUCTION}

Late Quaternary paleoclimates have been extensively studied across Africa including numerous sites in Western and Equatorial Africa. Details of earlier works on the Quaternary paleoclimates of Africa are contained in Sowunmi (1981b). Notably, more recent works include those of Talbot \& Delibrias (1980), Sowunmi (1981a, 1981b, 1986, 1987), Lezine (1989), Maley $(1991,1996)$, Maley \& Brenac (1998), Salzmann \& Waller (1998), Gasse (2000), Salzmann (2000), DeMenocal et al. (2000), Zabel et al. (2001), Tossou (2002), Adegbie et al (2003), Sowunmi (2004), and Orijemie \& Sowunmi (2014), Miller \& Gosling (2014) as well as Armitage et al. (2015). Sedimentary sequences for most of these earlier works were from marine and lake deposits with only a few from alluvial and coastal freshwater swamp areas. The instability of the coastal areas due to erosion, re-deposition, long-shore drifts, tidal movement etc., have limited their use in paleoenvironmental studies compared with the undisturbed layers of lake and deep marine sediments. However, Miller \& Gosling (2014) reiterated three 
important limitations of deep marine cores as Degroot and Degroot 1960 had earlier pointed out: i) dual pollen and spore transport media (wind and river), ii) complexity of the oceanic transport pathway with offshore water currents, and iii) low pollen content. They favored the use of lake sediments that have a clear sediment source area unlike deep marine ones with a broadly less defined source area. However, natural lakes are scarce in West Africa except for lakes Baronmbi-Mbo (Cameroun), Bosumtwi (Ghana) and Tilla (Chad). Besides, retrieving deep marine cores is a big challenge and needs international projects sponsored by international scientific drilling programs. Following the strategy of Sowunmi $(1981 a, 1987,2004)$ in which mangrove creek and freshwater sediments provided a convincing history of vegetational changes and paleoclimates of the Late Quaternary (last 35,000 years BP) in two Nigerian basins - the Niger delta and Benin Basin - a palynological and sedimentological study using coastal freshwater swamp sediments from Lagos, Nigeria in the eastern Benin Basin was undertaken. The aim of this study is to investigate the paleoclimatic changes of the Late Quaternary as recorded in the coastal deposits of the Benin Basin using palynomorphs, diatoms, and sedimentological parameters so as to infer its paleoecology.

\section{LAGOS STATE - CLIMATE, VEGETATION AND GEOLOGY}

Lagos State lies at latitude $\mathrm{N} 06^{\circ} 31.007$ and longitude $\mathrm{E} 003^{\circ} 23.936$ (Fig. 1) and it has a humid tropical climate with distinct dry and wet seasons with a maximum temperature range between $29^{\circ} \mathrm{C}$ and $34^{\circ} \mathrm{C}$, the lowest in July and the highest in February. Minimum temperature varies between $24^{\circ} \mathrm{C}$ and $28^{\circ} \mathrm{C}$. Mean annual rainfall varies between $1381.7 \mathrm{~mm}$ and $2733.4 \mathrm{~mm}$ while relative humidity is rarely below $70 \%$ throughout the year (Longe et al. 1987). There are hardly any months without rain. Two main seasons - dry and wet - with two shorter ones within, have been recognized for southwestern Nigeria and this area in Lagos (Adeniyi et al. 2009). They are the dry season (November - March), wet season (April - July), short dry season (late July - August) and the short wet season (September - October). The environment is coastal with wetlands, sandy barrier islands, beaches, low-lying tidal flats and estuaries (Jones \& Hockey 1964). Lagos is located in the coastal part of Nigeria and its vegetation is made up of four types, namely lowland rainforest, freshwater swamp forest, mangrove swamp forest, and beach vegetation (Fig. 2).

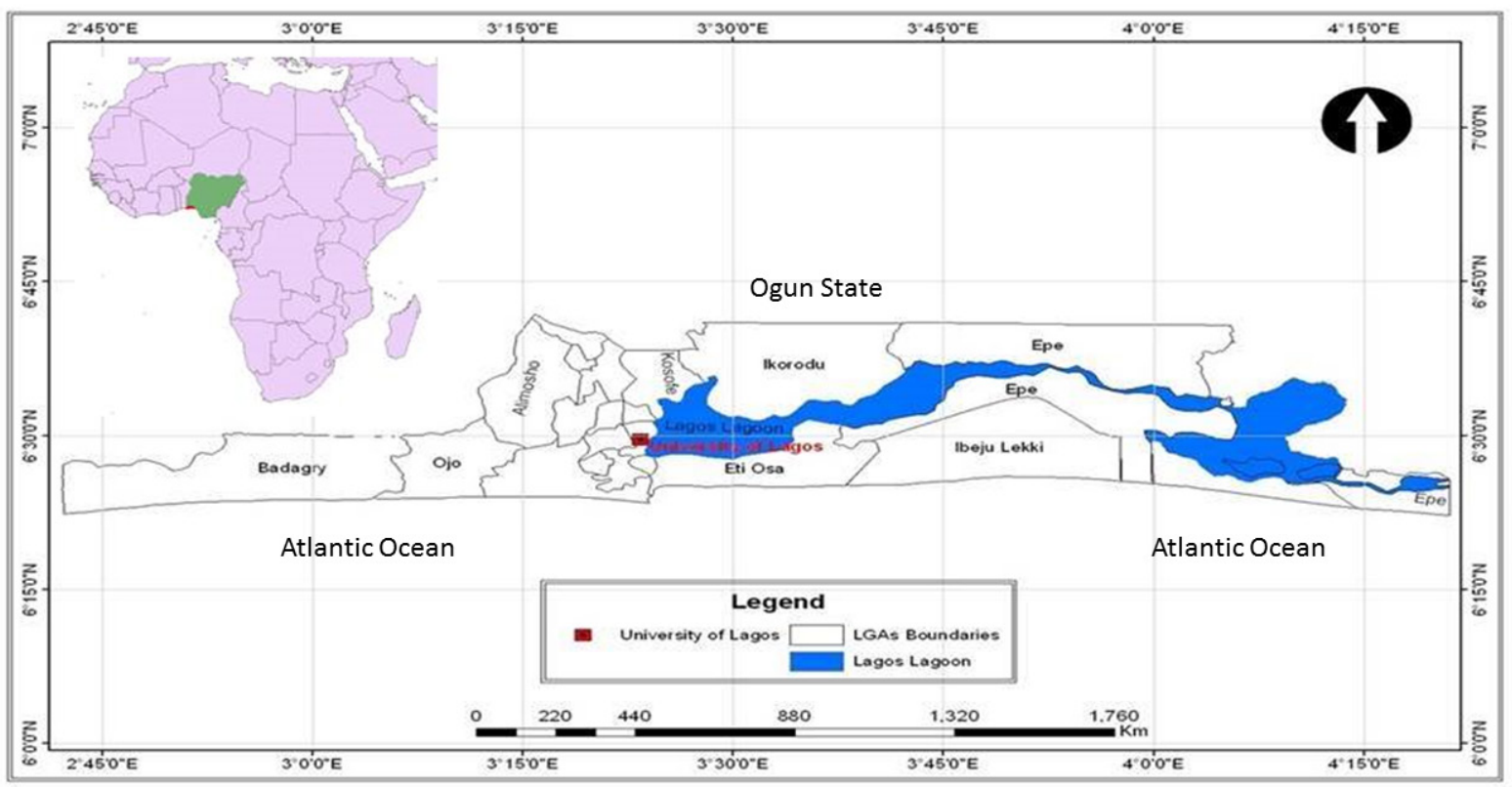

Fig. 1. Map of Lagos State highlighting the site of the UniLag Borehole at Akoka, Lagos 


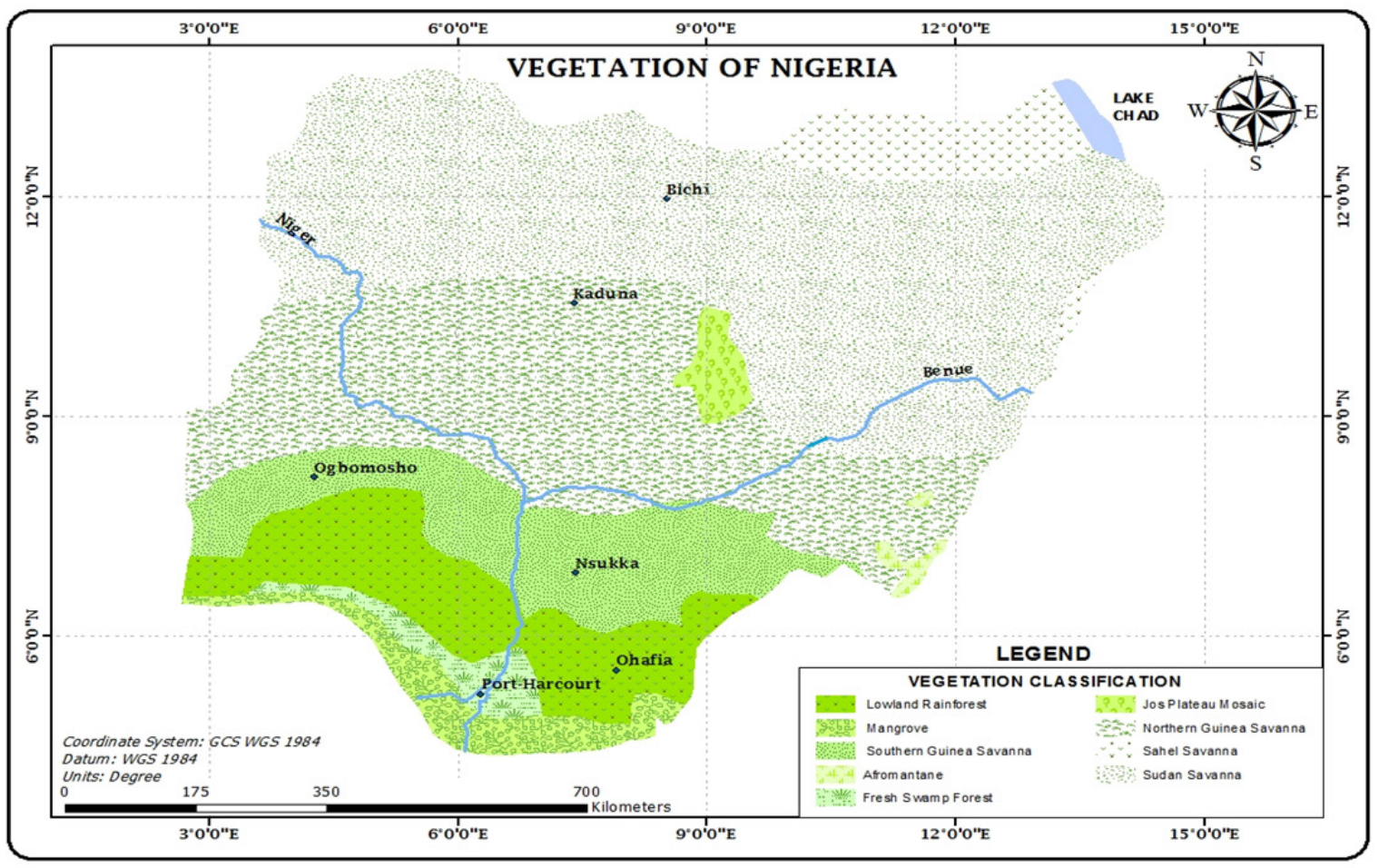

Fig. 2. Vegetation map of Nigeria

The Beach vegetation contains plants such as Ipomoea, Dalbergia, Sporobolus, Eugenia, Hibiscus, Phoenix and Barteri as found in all typical coastal areas of Nigeria (Keay 1959). In the brackish water area, the Mangrove Swamp vegetation is made up of Rhizophora spp. mainly, in areas south of Badagry Creek and reaching the Lagos Lagoon with abundant Raphia palms. Two strongly zoned species of Rhizophora make up its mangrove: red mangrove $R$. mangle (the saltier type) and black mangrove $R$. racemosa (less salty type - in the upper inland). Other members of the mangrove swamp include Avicennia germinans, Laguncularia racemosa, Drepanocarpus lunatus and the salt-water fern, Acrostichum aureum as well as the grass Paspalum spp. (Adekanmbi et al. 2009). The Freshwater Swamp vegetation type contains taxa such as Pandanus candelabrum, Podococcus barteri, Ancistrophylum opacum, Raphia spp., Bambusa vulgaris, Typha australis, and floating and sub-merged grasses (Paspalum, Panicum and Andropogon) and sedges (Cyperus, Kyllinga, Rhynchospora corymbosa and Mariscus) as well as typical freshwater forest trees such as Symphonia globulifera, Anthocleista, Hallea cilliata, Uapaca spp., Phoenix reclinata with shrubs, epiphytes and ferns (Nephrolepis and Dryopteris mainly). The Lowland Rainforest has tall trees such as Afromosia laxiflora, Azadiracta indica, Casuarina equisetifolia, Terminalia catappa, Alstonia boonei, Anthocleista spp. and Burkea africana. Some parts have metamorphosed into secondary forest due to recent developmental projects and cultivation. This secondary forest is dominated by oil palms and few trees, as well as shrubs such as Alchornea cordifolia, Gmelina arborea and Gliricidia sepium. Typical weeds in such cultivated parts are Chromolaena odorata, Panicum maximum and Sida acuta. The Lagos coastal area is located within the south eastern part of the Benin Basin (Dahomey Embayment) which is pseudo-parallel to the West African coast. It stretches from the Ghana Ridge onshore and extends through Togo and Benin Republics to the Benin Hinge Line in western Nigeria (Okosun 1990). It was formed in response to the separation of the African and South American landmasses with the subsequent opening of the Atlantic Ocean in the Jurassic to Lower Cretaceous (Omatsola \& Adegoke 1981, Coker \& Ejedawe 1987). Two structural 
units, the Benin Basin proper and the Okitipupa structure were recognized by Coker \& Ejedawe (1987). Commencing from the Ghana Ridge to the Orimedu-Ijebu Ode - Ilorin axis including Lagos is the Benin Basin proper, while the Okitipupa Structure stretches from the Ijebu Ode - Ilorin axis to the Benin Hinge Line. The Benin Basin proper shows a long strike, a steady drop in the basement floor from east to west and a north to south steep profile ranging from 1900-2200 m and $1513-2727 \mathrm{~m}$ respectively. Except for the Ise graben (1800-2000 m) the Okitipupa structure, however, indicates a shallower basement floor ranging between 1000-1400 m (Coker \& Ejedawe 1987). In the Lower Cretaceous (Neocomian), a thick sequence of continental grits and pebbly sands $(>1400 \mathrm{~m})$ were deposited across the entire basin due to rift-generated basement subsidence (Omatsola \& Adegoke 1981). Following this was a tectonic event in the Santonian which is linked to the closure and folding of the Benue Trough that resulted in the tilting and block faulting of granites, gneisses and associated pegmitites and other sediments to form a series of grabens. Accompanying this tectonic activity was considerable erosion which eroded the pre-drift sediments greatly from the horsts (Omatsola \& Adegoke 1981). The stratigraphy of this basin is made up of the Abeokuta, Ewekoro, Akinbo, Oshoshun, Ilaro and Benin formations straddling the Neocomian to Recent. The Abeokuta Formation sits conformably on the basement complex. It is Neocomian/ Paleocene in age and has been assigned a group status and sub-divided by Omatsola \& Adegoke (1981) into three formations - Ise, (oldest); Afowo; and Araromi (youngest). The Paleocene/Eocene limestone, marine shales and sandy shales, and claystones of Ewekoro, Oshosun, and Ilaro Formations respectively sit conformably on top of the Abeokuta Formation. Sitting unconformably on and capping the Paleocene/Eocene stratigraphic sequence is the Late Tertiary sediments of Benin Formation with shallow marine - none marine gravel, sand and sandy clay (Ogbe 1972, Omatsola \& Adegoke 1981) (Tab. 1). Billman (1992) reported that a thick sequence of the Benin Basin in the offshore Benin Republic is Pre-Albian to Late Miocene.
Table 1

Stratigraphy of Benin Basin

\begin{tabular}{|l|l|l|}
\hline \multicolumn{1}{|c|}{ Age } & $\begin{array}{c}\text { Formation } \\
\text { (Ogbe 1972) }\end{array}$ & $\begin{array}{c}\text { Formation } \\
\text { (Omatsola \& } \\
\text { Adegoke 1981) }\end{array}$ \\
\hline $\begin{array}{l}\text { Middle Miocene- } \\
\text { Recent }\end{array}$ & Benin & Benin \\
\hline Lower Eocene & Ilaro & Ilaro \\
\hline Lower Eocene & Oshoshun & Oshoshun \\
\hline Paleocene & Akinbo & Oshoshun \\
\hline Paleocene & Ewekoro & Ewekoro \\
\hline Senonian-Paleocene & Abeokuta & Araromi \\
\hline Senonian & Abeokuta & Afowo \\
\hline Neocomian-Senonian & Abeokuta & Ise \\
\hline & & \\
\hline
\end{tabular}

\section{DESCRIPTION OF THE SITE}

The borehole location in the University of Lagos, Akoka campus, Lagos falls between latitude $\mathrm{N} 06^{\circ} 31.007$ and longitude E $003^{\circ} 23.936$ (Fig. 1). The present vegetation of the borehole site is predominantly the open secondary freshwater forest type with tree taxa such as Anthocleista spp. Elaeis guineensis, Terminalia catappa and Musa spp. Characteristic herbs and shrubs which predominate in the site are Tridax procumbens, Panicum maximum, Ipomoea sp., Alchornea cordifolia, Mimosa pudica, Luffa cylindrical, Borreria sp. and Amaranthaceae. Close-by relics of mangrove taxa about $80 \mathrm{~m}$ away include Rhizophora spp., $L a$ guncularia racemosa, and Avicennia germinans. Also abundant around this site were Drepanocarpus lunatus and Acrostichum aureum. Other taxa present in minor proportions are Triumffeta sp., Gomphrena celosoides, Cyperus sp., Oldenlandia affrinis, Ipomoea carica, Cnestia penuginum, Alternatera sessilis, Centrocina pubescens, Cyathula prostrata, Phycus spp., Panicum sp., Phyllanthus reticulatus, Diodia scadense, Manihot esculanta, Dalbergia ecastaphyllum, Vernonia cinera, Zea mays, Mariscus alternifolius, Spigelia anthelma, Sacciolepis africana, Emilia coccinea, Scoparia dulcis, Solenostemon polystachon, Andropogon spp., and Azadirachta indica.

\section{MATERIALS AND METHODS}

A total of sixteen borehole samples made available by Automated Geotechnics Ltd. Lagos, Nigeria 
were used. The samples were collected at $2 \mathrm{~m}$ intervals for palynological and sedimentological analyses. The 16 sub-samples were subjected to standard palynological treatment involving $\mathrm{HCl}$, $\mathrm{HF}$, and acidified $\mathrm{ZnCl}_{2}$ solution with acetolysis according to Erdtman (1969). Residues were put in calibrated test tubes and a known volume of $100 \%$ glycerine was added to estimate the residue volume. Micro-slides were prepared for quantitative and qualitative microscopic analysis using an Olympus light microscope ( $\times 40$ objective lens). Identification of recovered palynomorphs was made through published atlases and journals such as Sowunmi (1973, 1995), Gosling et al. (2013) and the reference slide collection of the Paleobotany/ Palynology Laboratory, Department of Botany, University of Lagos, Nigeria. Unidentified pollen and spores were categorized as Indeterminates and excluded from the Pollen Sum but considered in the percentage calculation. Pollen sum for all the levels were calculated involving Rhizophora spp. and Poaceae since they dominate only occasionally as observed from the analyzed slides. Using the recovered pollen and spores, eight phytoecological groups were made on the basis of the known present day natural distribution of their parent plant sources. The works of Hutchinson \& Dalziel (1954, 1958, 1963, 1968, 1972), Keay (1959), Sowunmi (1973, 1995, 1981a, 1981b), Adeonipekun (2006) and Adeonipekun et al. (2015) guided the grouping.

For the sedimentological analysis, sub-samples were gently crushed and washed with water until they became clean and dried for microscopic analysis through visual examination using the American/Canadian stratigraphic code. The roundness, color, sphericity and sorting of the sand particles were recorded and the occurrence and non-occurrence of accessory minerals such as ferruginous materials, glauconites, carbonaceous detritus, pyrites, mica flakes and dolomite grains as well as the in features of shale particles were also noted for each appropriate depth (Selley 1976). For salinity measurements, a refractometer (Salinometer) was used and the salinity value of each sub-sample was read and noted. Age control for the analyzed sediments was achieved by Accelerator Mass Spectrometry (AMS) radiocarbon dating of the organic matter at Radiochronology Laboratory
C.E.N. (Centre for Northern Studies) Université Laval, Abitibi-Price, Quebec, Canada and Center for Applied Isotope Studies, University of Georgia, Athens USA. Two samples from depths of 6-12 m were radiocarbon dated. None of the samples below $12 \mathrm{~m}$ depth had enough organic matter for radiocarbon dating hence extrapolation was done for the ages of events in this lower section.

\section{RESULTS}

Categorizing the phytoecological groups into dry and wet paleoclimate groups resulted in having Lowland rainforest, Mangrove, Spores, and Freshwater swamp forest types belonging to the Wet Paleoclimate Indicator (WPI) group while the Dry Paleoclimate Indicator (DPI) group consists of Poaceae, Savanna, Open vegetation and Montane vegetation types. Figures 3-6 show the relationship between the wet and dry paleoclimate indicator taxa as well as phytoecological groups and their component taxa. Fungal hyphae and spores, Concentricytes, freshwater Botryococcus and Pediastrum as well as a few dinoflagellates along with some diatom taxa that survived the HF treatment are also shown in Figures 6A, B.

Ten sediment units that correspond to ten lithological types were recognized in the $30 \mathrm{~m}$ long sequence (Tabs. 2-4). They are described as follows:

- lithofacies type 1: coarse, sub-angular to sub-rounded sand,

- lithofacies type 2: blocky shale,

- lithofacies type 3: hard shale / mudstones,

- lithofacies type 4: mixture of medium-grained sand and silty shale,

- lithofacies type 5: sandy clay with poorly sorted sand,

- lithofacies type 6: dolomitic mudstone with abundant amorphous organic matter,

- lithofacies type 7: dolomitic hard shale with abundant humic gel and brown wood,

- lithofacies type 8: dolomitic clay with pockets of well sorted sands and abundant brown woods,

- lithofacies type 9: composed of creamy white moderate to well sorted sands,

- lithofacies type 10: composed of cream white well sorted sands. 


\begin{tabular}{|c|c|c|c|c|c|c|c|c|c|c|c|c|c|c|c|c|}
\hline 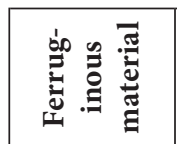 & + & + & 1 & 1 & 1 & + & + & + & + & 1 & + & + & 1 & + & 1 & 1 \\
\hline 总 & 1 & 1 & $\mid \begin{array}{l}\vec{z} \\
\vec{z} \\
\vec{z}\end{array}$ & 莺 & 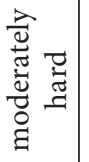 & ت্ট్ & 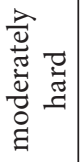 & 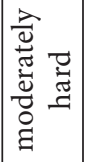 & 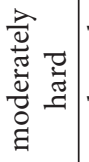 & 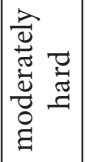 & 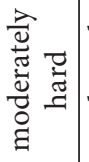 & 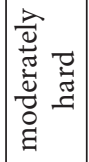 & 1 & 1 & 1 & 1 \\
\hline 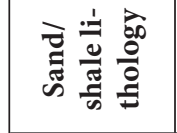 & 1 & 1 & 1 & $\begin{array}{l}\stackrel{n}{n} \\
\dot{i n}\end{array}$ & $\begin{array}{l}\tilde{\infty} \\
\dot{\infty}\end{array}$ & $\begin{array}{l}\tilde{\alpha} \\
\dot{\alpha}\end{array}$ & 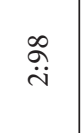 & 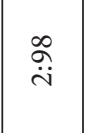 & 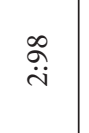 & 1 & 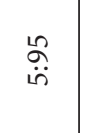 & $\begin{array}{c}\infty \\
\stackrel{i}{i}\end{array}$ & 1 & 1 & 1 & 1 \\
\hline 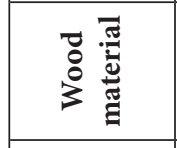 & 1 & 1 & 1 & 1 & + & 1 & 1 & 1 & 1 & 1 & 1 & + & + & + & 1 & 1 \\
\hline 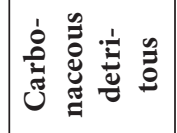 & + & + & + & + & 1 & + & + & + & + & 1 & 1 & + & 1 & 1 & 1 & 1 \\
\hline 荧蒫 & 1 & 1 & 1 & 1 & 1 & 1 & 1 & 1 & 1 & 1 & & + & + & + & + & 1 \\
\hline 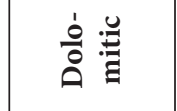 & 1 & 1 & 1 & 1 & 1 & 1 & 1 & 1 & + & + & + & 1 & 1 & 1 & 1 & 1 \\
\hline 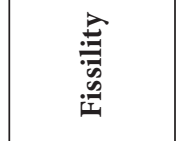 & 1 & 1 & $\mid \begin{array}{l}\frac{2}{4} \\
\frac{0}{0} \\
\frac{0}{3}\end{array}$ & $\begin{array}{l}\frac{\hat{y}}{0} \\
0 \\
0\end{array}$ & 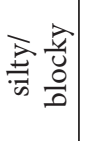 & 1 & 1 & 1 & $\begin{array}{l}\frac{\hat{y}}{0} \\
\frac{0}{b}\end{array}$ & $\begin{array}{l}\frac{\hat{y}}{\mathrm{c}} \\
\frac{0}{0}\end{array}$ & $\begin{array}{l}\frac{\hat{y}}{0} \\
\frac{0}{0}\end{array}$ & 1 & 1 & 1 & 1 & 1 \\
\hline 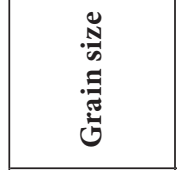 & 苞 & 苞 & 1 & 1 & 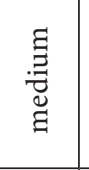 & 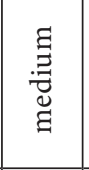 & 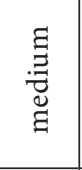 & 1 & 1 & 1 & 节 & 节 & 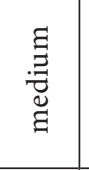 & 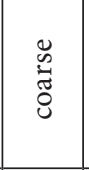 & 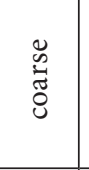 & 节 \\
\hline 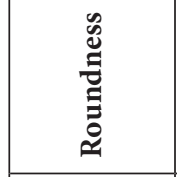 & 它 & 它 & 1 & 1 & 它 & 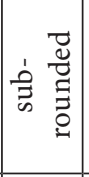 & 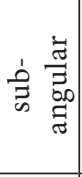 & 1 & 1 & 1 & 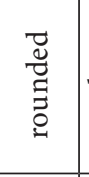 & 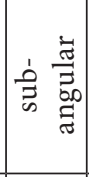 & 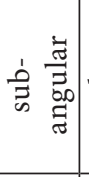 & 完 & 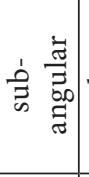 & 官 \\
\hline 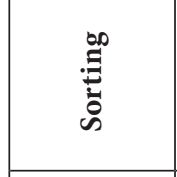 & 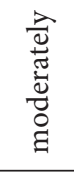 & 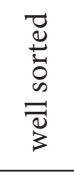 & 1 & 1 & 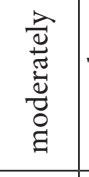 & 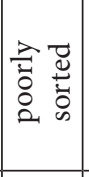 & 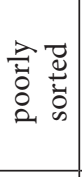 & 1 & 1 & 1 & $\begin{array}{l}\vec{\Xi} \\
\stackrel{\Xi}{0} \\
0 \\
\overrightarrow{0} \\
3\end{array}$ & $\begin{array}{l}\overrightarrow{0} \\
\stackrel{0}{0} \\
0 \\
\overline{0} \\
\overline{0} \\
3\end{array}$ & 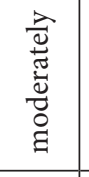 & 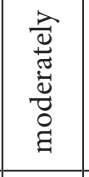 & 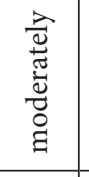 & 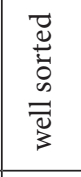 \\
\hline$\frac{3}{0}$ & 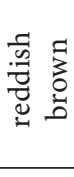 & 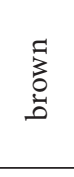 & 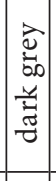 & 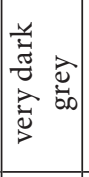 & 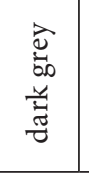 & 㫄 & 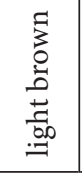 & है & 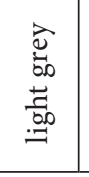 & 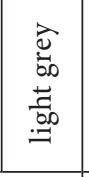 & 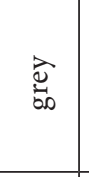 & 氦 & 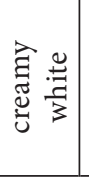 & 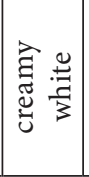 & 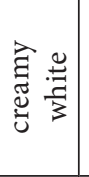 & $\begin{array}{l}\text { हี } \\
\text { ֻँّ }\end{array}$ \\
\hline 象象 & \multicolumn{2}{|c|}{-} & $\sim$ & $m$ & \multicolumn{2}{|c|}{$r$} & \multicolumn{2}{|c|}{ in } & \multicolumn{2}{|c|}{0} & $\wedge$ & $\infty$ & \multicolumn{3}{|c|}{$a$} & 음 \\
\hline 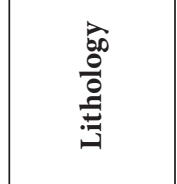 & 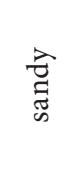 & 氛 & $\frac{\omega}{\pi}$ & 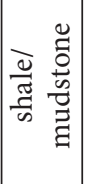 & 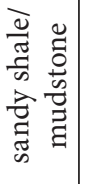 & 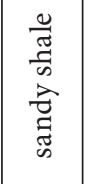 & 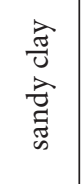 & 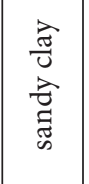 & $\begin{array}{l}\tilde{\Xi} \\
\tilde{0} \\
\tilde{0} \\
\tilde{\Xi} \\
\Xi\end{array}$ & 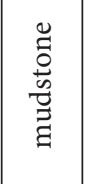 & 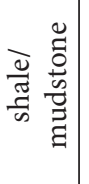 & 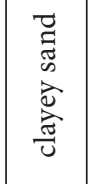 & 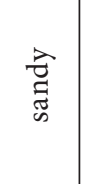 & 预 & 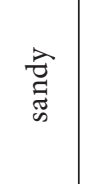 & 鄢 \\
\hline 竞目 & $\stackrel{0}{\circ}$ & $\stackrel{\text { ì }}{\text { to }}$ & $\stackrel{+}{+}$ & : & $\stackrel{0}{\infty}$ & $\stackrel{\leftrightarrow}{\varrho}$ & $\stackrel{\circ}{\mathrm{I}}$ & $\stackrel{\text { I }}{\stackrel{\circ}{ }}$ & : & 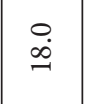 & $\ddot{\text { iे }}$ & ì & $\stackrel{\text { İ }}{\mathrm{i}}$ & : & $\stackrel{\infty}{\stackrel{\infty}{\sim}}$ & $\stackrel{\dot{m}}{\circ}$ \\
\hline
\end{tabular}


Table 3

Palynofacies and lithofacies of UniLag Borehole

\begin{tabular}{|c|c|c|c|}
\hline Period & $\begin{array}{l}\text { Depth } \\
{[\mathrm{m}]}\end{array}$ & Palynofacies & $\begin{array}{l}\text { Litho- } \\
\text { facies }\end{array}$ \\
\hline \multirow{16}{*}{$\begin{array}{l}\text { Late } \\
\text { Quaternary }\end{array}$} & 0.0 & $\begin{array}{l}80 \% \text { particulate AOM, humic gel and degraded plant materials } 20 \% \text {, sub-angular to } \\
\text { rounded } 80 \% \text { GM }\end{array}$ & \multirow[t]{2}{*}{1} \\
\hline & 2.0 & $50 \%$ PAOM, 50\% GM & \\
\hline & 4.0 & $\begin{array}{l}\text { Large, greenish yellow, angular, woody with cuticles and parenchyma cells. Poor humic } \\
\text { gel with } 70 \% \text { GM }\end{array}$ & 2 \\
\hline & 6.0 & Poor humic gel, angular to sub-angular greenish woods with 35\% GM & 3 \\
\hline & 8.0 & Poor wood, greenish yellow plant materials, no humic gel with $20 \%$ GM & \multirow[b]{2}{*}{4} \\
\hline & 10.0 & $\begin{array}{l}\text { High cuticle, 25\% GM, woody and plant materials angular to sub-angular and greenish } \\
\text { yellow. Poor humic gel }\end{array}$ & \\
\hline & 12.0 & No wood, humic gel and few degraded plant materials, PAOM. Greenish black & \multirow{2}{*}{5} \\
\hline & 14.0 & Brown wood, degraded plant materials, AOM present, sub-rounded to rounded, GM 20\% & \\
\hline & 16.0 & $\begin{array}{l}\text { AOM } 90 \% \text { and humic gel dominate with few degraded plant materials. Poor sorting, large } \\
\text { to small, rounded to sub-angular GM } 40 \%\end{array}$ & \multirow[t]{2}{*}{6} \\
\hline & 18.0 & AOM $100 \%$, few humic gel and occasional yellow plant material & \\
\hline & 20.0 & Large humic gel, AOM, brown wood dominate, angular to sub-angular with $10 \% \mathrm{GM}$ & 7 \\
\hline & 22.0 & Brown wood dominates with humic gel and AOM following, angular. GM 10\% & 8 \\
\hline & 24.0 & \multirow{2}{*}{$\begin{array}{l}\text { Humic gel dominates with few brown woods. Poor GM less than 5\%, angular to sub- } \\
\text { angular debris }\end{array}$} & \multirow{2}{*}{9} \\
\hline & 26.0 & & \\
\hline & 28.0 & \multirow{2}{*}{ High humic gel and brown wood with $10 \%$ GM. Angular to sub-rounded } & \multirow{2}{*}{10} \\
\hline & 30.0 & & \\
\hline
\end{tabular}

Table 4

Radiocarbon dates of two samples from UniLag Borehole sediments

\begin{tabular}{|c|c|c|c|c|c|c|}
\hline University of Georgia & Université Laval & Depth $[\mathbf{m}]$ & pMC & \pm & ${ }^{14}$ C age $[$ BP] & \pm \\
\hline UGAMS 21399 & ULA-5375 & 6.00 & 50.47 & 0.16 & 5,495 & 25 \\
\hline UGAMS 21404 & ULA-5376 & 12.00 & 14.24 & 0.08 & 15,660 & 40 \\
\hline
\end{tabular}

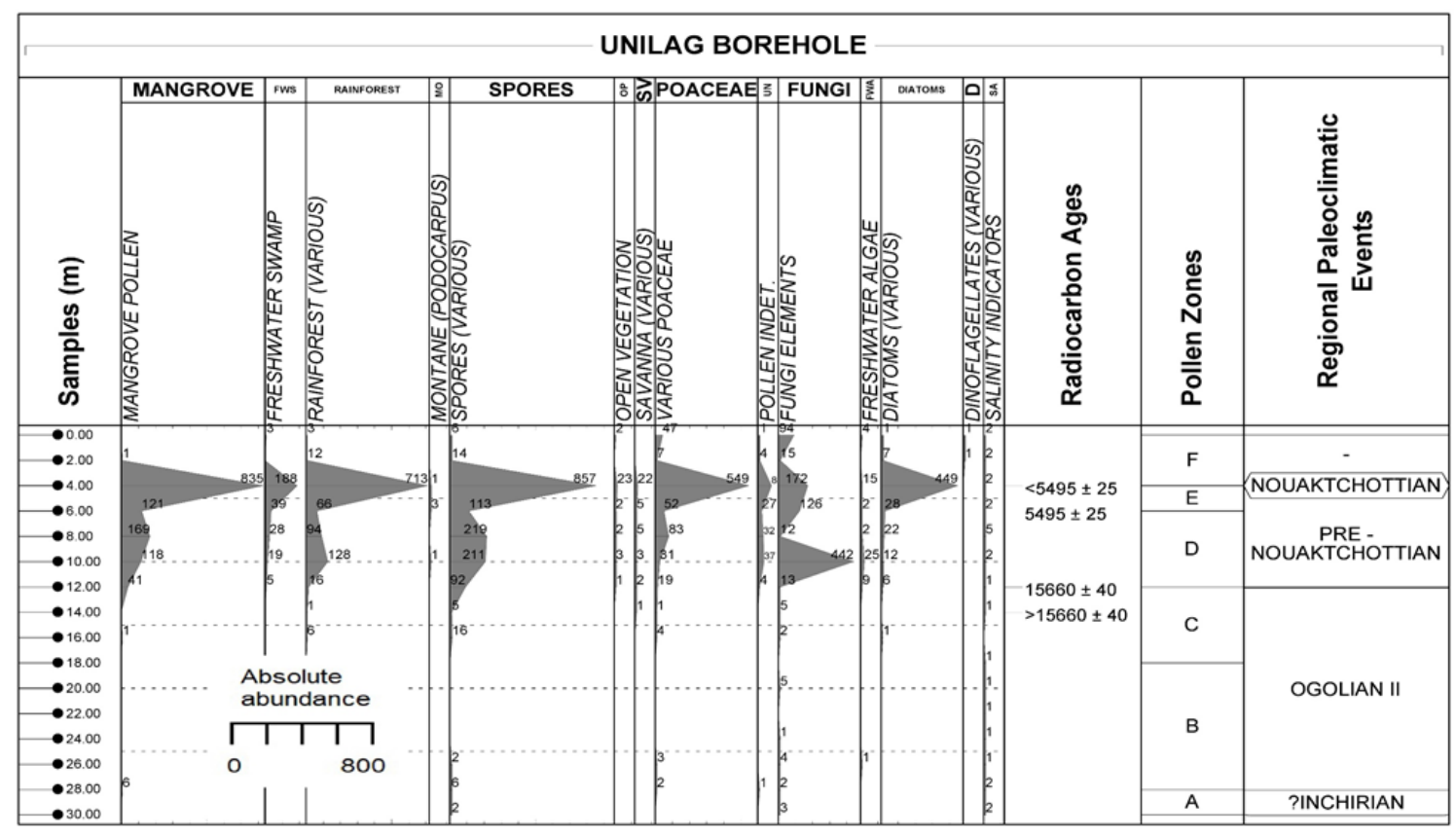

Fig. 3. Phytoecological groups, accessory palynomorphs, diatoms and salinity values of the UniLag Borehole sediments 


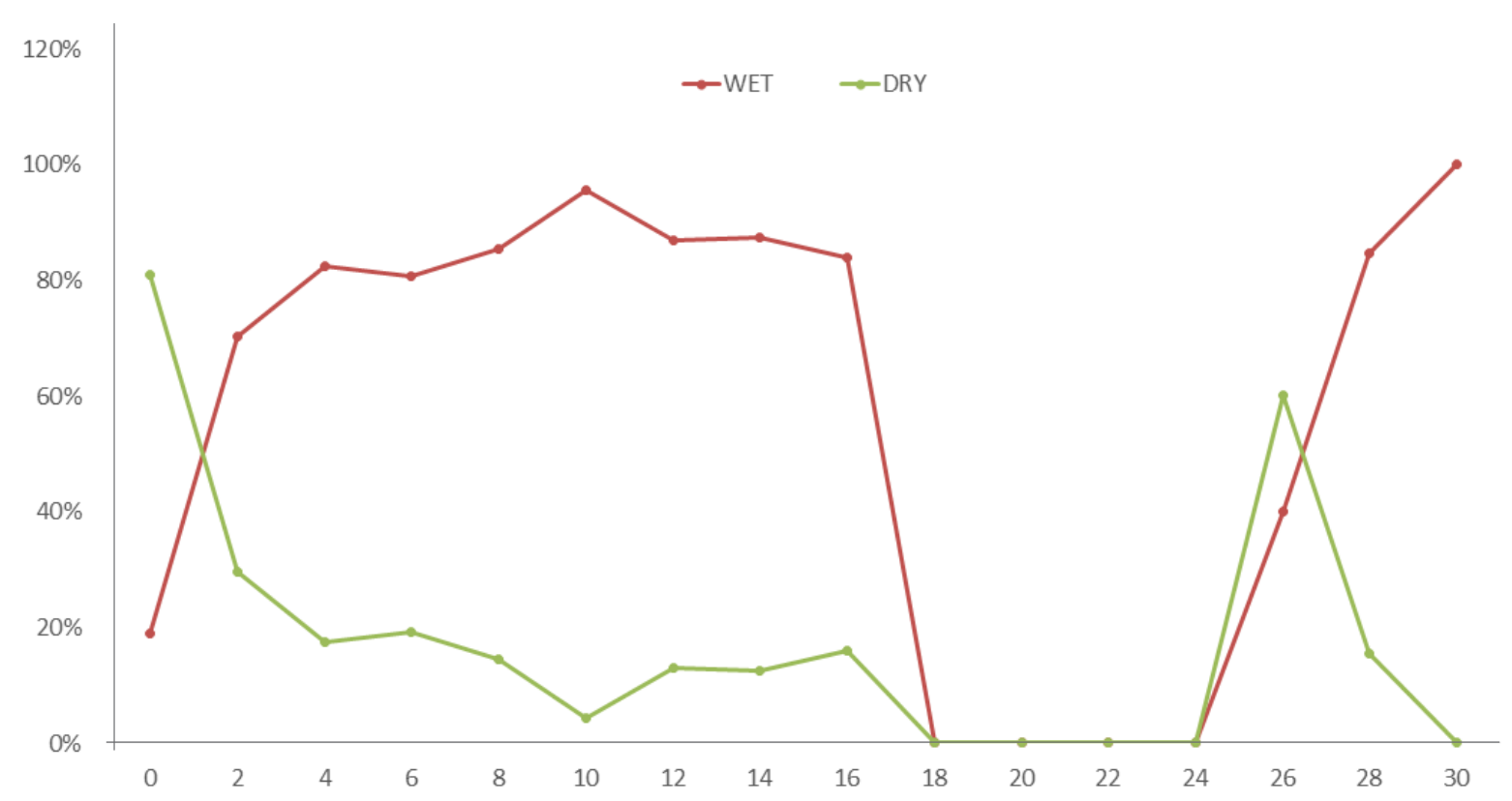

Fig. 4. Percentage abundance values of WPI and DPI against depth [m]

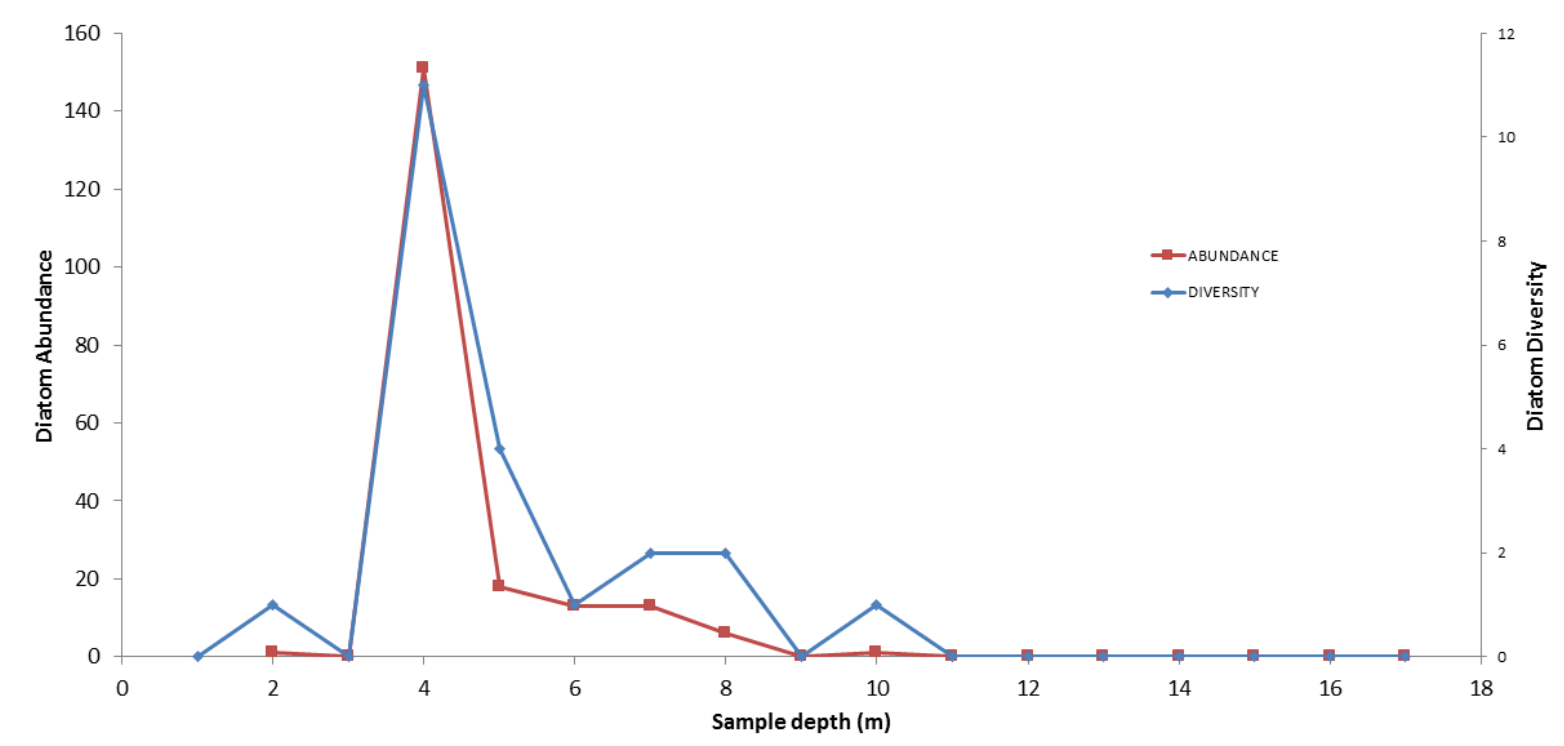

Fig. 5. Diatom diversity/abundance/depth $[\mathrm{m}]$ of the UniLag Borehole

Six pollen zones (A-F) were identified based on the phytoecological, and paleoclimatic inferences (Tab. 5). The pollen zones represent two dry phases (represented by pollen zones B and F), and two wet phases (A and D), one very wet/cold phase (E pollen zone) and one transition phase (C pollen zone). The very wet/cold zone $\mathrm{E}$ with an unprecedented abundance of Mangrove and all other groups including diatoms and freshwater Botryococcus and Pediastrum is of great regional paleoclimatic significance. The inference of coldness stems from the relatively highest values of Podocarpus milanjianus pollen recovered within this zone. Zone B corresponds to dolomitic lithofacies and is therefore devoid of palynomorphs. Very few charred graminoid cuticles were recorded only in the sample at $4 \mathrm{~m}$ depth. 
$\mathrm{A}$

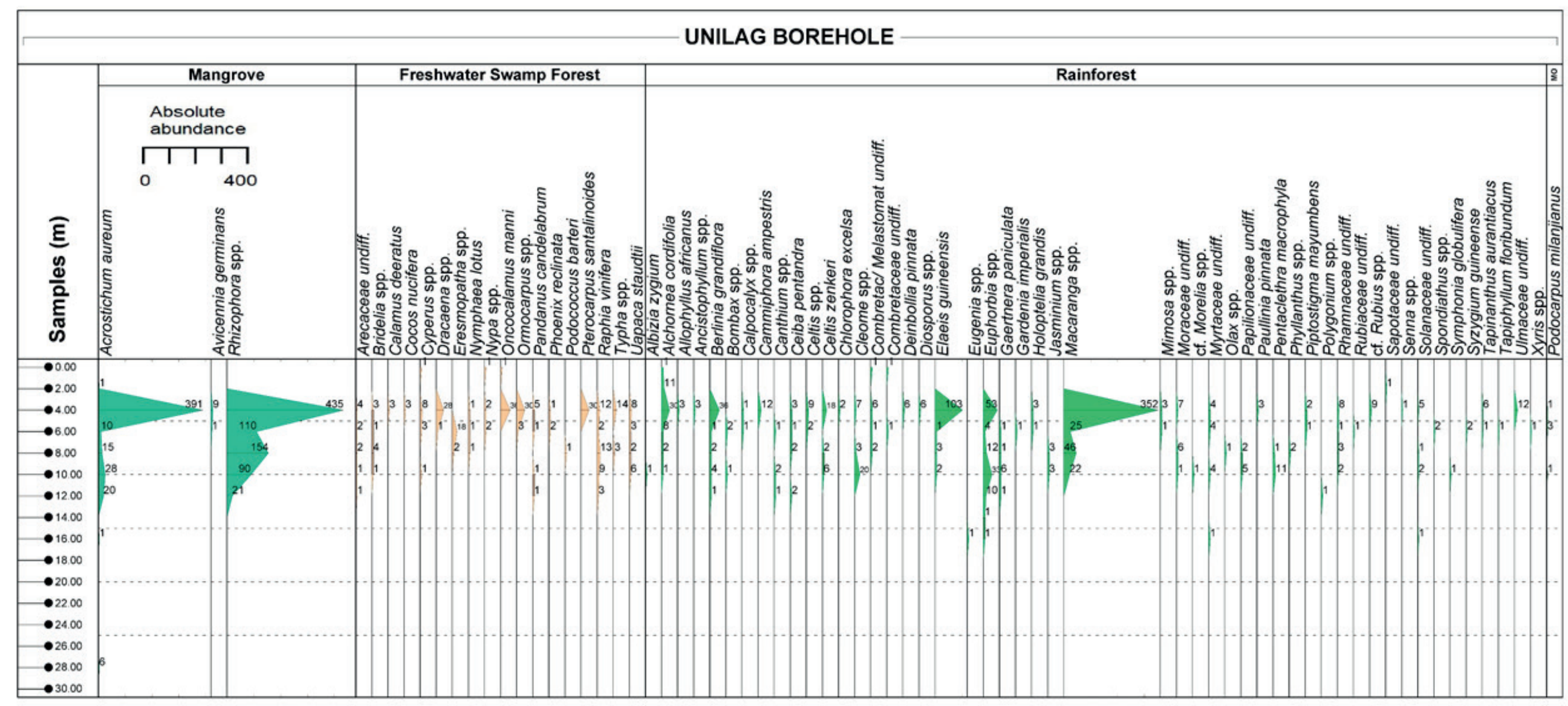

B

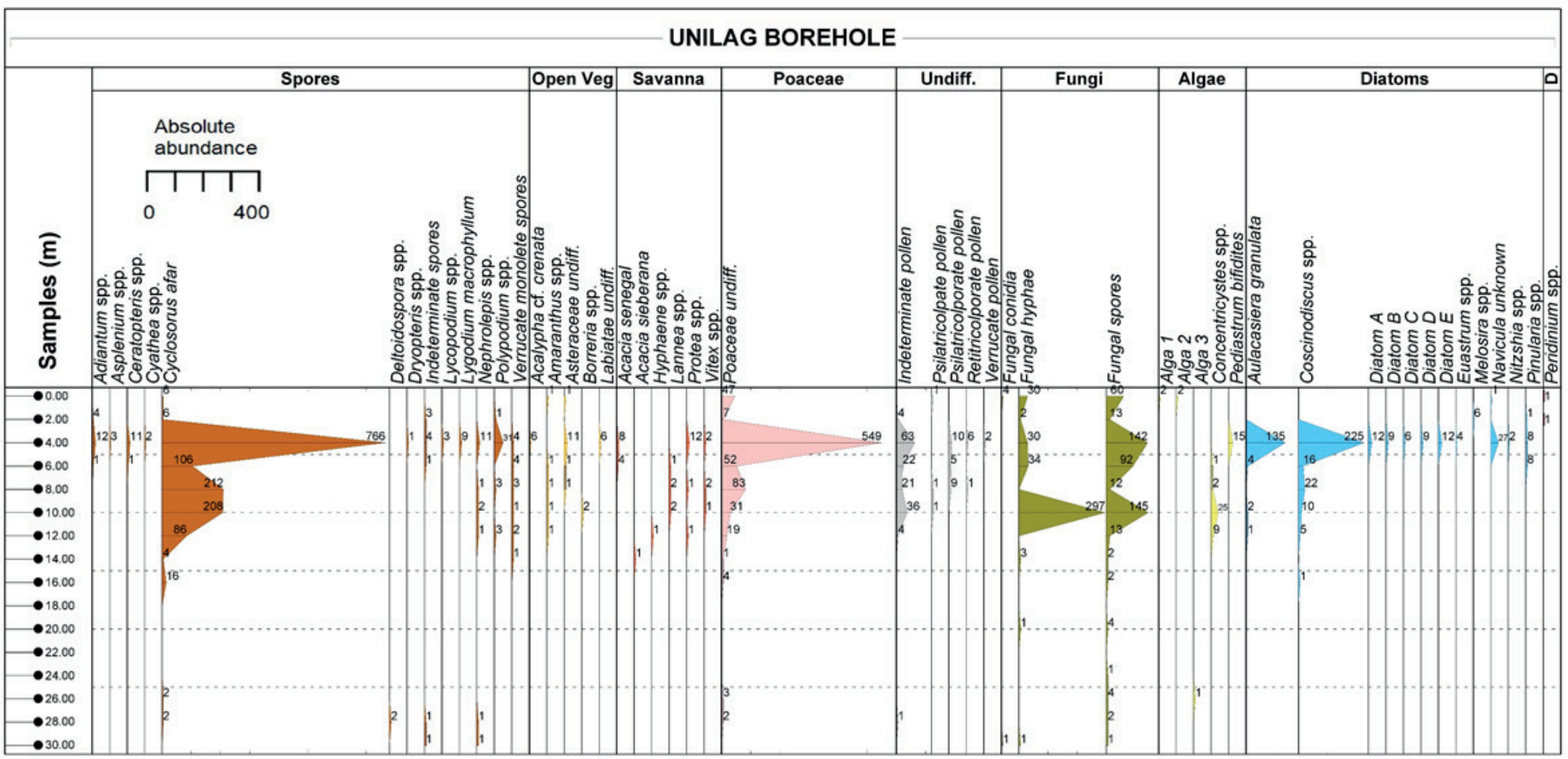

Fig. 6. Distribution chart of palynomorphs and diatoms of the UniLag Borehole sediments (A and B) 


\begin{tabular}{|c|c|c|c|c|c|c|c|c|c|c|c|c|c|c|c|c|}
\hline 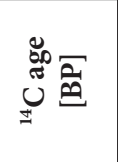 & & & & $\begin{array}{l}20 \\
+1 \\
+1 \\
2 \\
2 \\
+1 \\
i n\end{array}$ & & & $\begin{array}{l}+1 \\
+1 \\
\text { D: } \\
\text { : } \\
\text { L }\end{array}$ & & & & & & & & & \\
\hline 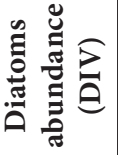 & $\Xi$ & @) & 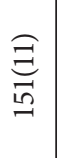 & 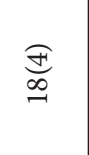 & $\underset{\sim}{\stackrel{\overbrace{}}{=}}$ & 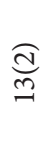 & $\widehat{\widehat{d}}$ & $\widehat{o}$ & $\Xi$ & $\stackrel{\varrho}{o}$ & $\widehat{\varrho}$ & oิ & $\widehat{\varrho}$ & $\stackrel{\varrho}{\circ}$ & $\widehat{\varrho}$ & $\widehat{\varrho}$ \\
\hline 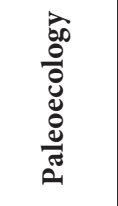 & \multicolumn{6}{|c|}{ 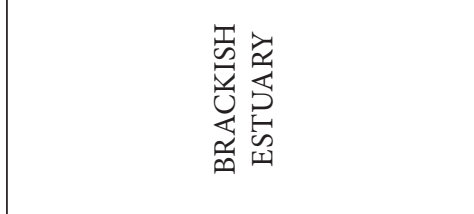 } & \multicolumn{8}{|c|}{ 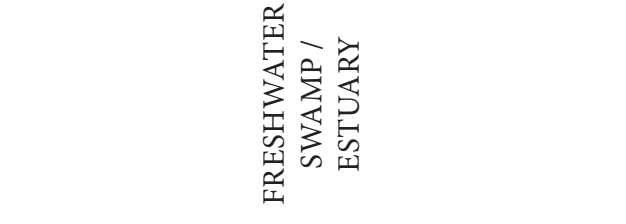 } & \multicolumn{2}{|c|}{ 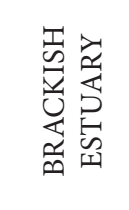 } \\
\hline 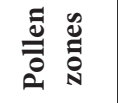 & \multicolumn{2}{|c|}{$\omega$} & 피 & \multicolumn{4}{|c|}{ A } & \multicolumn{2}{|c|}{$u$} & \multicolumn{5}{|c|}{$\infty$} & \multicolumn{2}{|c|}{$\varangle$} \\
\hline 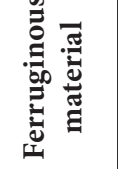 & + & + & 1 & 1 & 1 & + & + & + & + & 1 & + & + & 1 & + & 1 & 1 \\
\hline 产 & 1 & 1 & 1 & 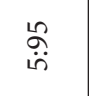 & $\begin{array}{l}\tilde{\alpha} \\
\dot{\infty}\end{array}$ & $\begin{array}{l}\widetilde{\alpha} \\
\dot{\infty}\end{array}$ & $\stackrel{\infty}{\stackrel{i}{i}}$ & $\stackrel{\infty}{\stackrel{i}{i}}$ & $\stackrel{\infty}{\stackrel{i}{i}}$ & & $\stackrel{\text { }}{\stackrel{\leftrightarrow}{i n}}$ & $\stackrel{\infty}{\grave{i}}$ & 1 & 1 & 1 & 1 \\
\hline 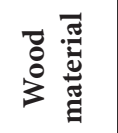 & 1 & 1 & 1 & 1 & + & 1 & 1 & 1 & 1 & 1 & 1 & + & + & + & 1 & 1 \\
\hline 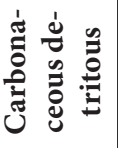 & + & + & + & + & 1 & + & + & + & + & & 1 & + & 1 & 1 & 1 & 1 \\
\hline 氬 & 1 & 1 & 1 & 1 & 1 & 1 & 1 & 1 & 1 & 1 & 1 & + & + & + & + & 1 \\
\hline$\frac{\vdots}{8}$ & 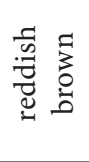 & 苟 & 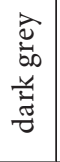 & 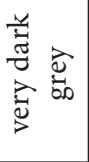 & $\begin{array}{l}\widehat{े} \\
\bar{b} \\
\breve{u} \\
\text { चै }\end{array}$ & مै & $\begin{array}{l}5 \\
3 \\
0 \\
0 \\
0 \\
\frac{0}{600} \\
:=\end{array}$ & 苟 & 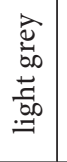 & 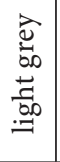 & ⿹े⿺ & $\begin{array}{l}\overrightarrow{\bar{v}} \\
\dot{b}\end{array}$ & 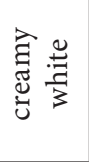 & 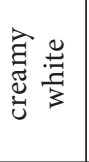 & 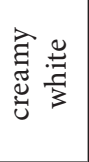 & 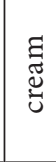 \\
\hline 总总 & \multicolumn{2}{|c|}{-} & $\sim$ & $m$ & \multicolumn{2}{|c|}{ H } & \multicolumn{2}{|c|}{ in } & \multicolumn{2}{|c|}{6} & $\wedge$ & $\infty$ & \multicolumn{3}{|c|}{$a$} & $\stackrel{\circ}{ }$ \\
\hline & $\begin{array}{l}\text { 矛 } \\
\text { 劳 }\end{array}$ & 帘 & $\frac{\frac{\omega}{\pi}}{\frac{\pi}{\infty}}$ & 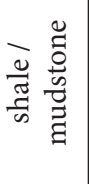 & 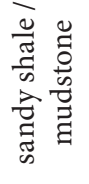 & 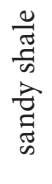 & 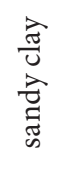 & 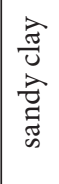 & 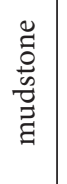 & 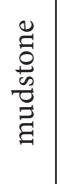 & 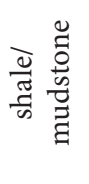 & 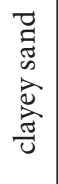 & 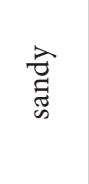 & 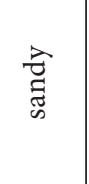 & 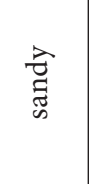 & 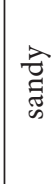 \\
\hline 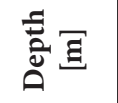 & $\stackrel{0}{\circ}$ & $\stackrel{\circ}{i}$ & $\stackrel{\circ}{+}$ & $\stackrel{0}{6}$ & $\stackrel{0}{\infty}$ & $\stackrel{\circ}{\circ}$ & $\stackrel{0}{\mathrm{I}}$ & $\stackrel{\circ}{\stackrel{+}{ \pm}}$ & $\stackrel{0}{\dot{\varphi}}$ & $\begin{array}{l}0 \\
\stackrel{\infty}{\sim}\end{array}$ & ¿̊ं & $\stackrel{\circ}{i}$ & $\stackrel{\circ}{i}$ & $\stackrel{0}{\dot{i}}$ & $\begin{array}{l}\circ \\
\dot{d} \\
\text { in }\end{array}$ & $\dot{m}$ \\
\hline 怤 $\bar{v}$ & $\stackrel{3}{-}$ & & & 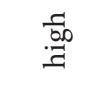 & & & s. & & & $\begin{array}{l}\overrightarrow{.0} \\
\overrightarrow{0.0}\end{array}$ & $\underline{3}$ & & 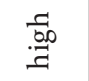 & ?3. & 点 & \\
\hline & & & H & & & & & $m$ & & & & $\sim$ & & & - & \\
\hline
\end{tabular}


Radiocarbon dates from organic matter of samples at $12 \mathrm{~m}$ and $6 \mathrm{~m}$ depths indicate that the $12 \mathrm{~m}$ depth sediments sample was deposited at $15,660 \pm 40{ }^{14} \mathrm{C}$ yr BP while the $6 \mathrm{~m}$ depth sediments were deposited at $5495 \pm 25{ }^{14} \mathrm{C}$ yr BP. Poor organic contents of the lower intervals however precluded their use for dating (Tab. 4). Four hydrological cycles with alternating high and low water levels each were identified for the approximately 35,000 yr BP borehole. These recognized pollen zones are described fully with respect to the paleoecological and paleoclimatic inferences below.

\section{DISCUSSION}

The palynological analysis revealed the paleo-vegetational changes that took place over time in the study area and indirectly reflect the paleoclimatic/ paleoecologic conditions. Pollen and spores of different ecological zones in Nigeria (forest, savanna and montane) were recovered. It is important to note that one single occurrence of a typical savanna pollen was found in one of the treated samples and hence makes up the Savanna group for its significance at that level. Sowunmi (1981a), Adeonipekun (2006) and Adeonipekun et al. (2015) reported low occurrences of typical savanna pollen from the Late Quaternary and Late Tertiary sediments respectively of the Niger delta relative to the rainforest and even airborne montane Podocarpus milanjianus pollen. This indicates that the Poaceae recorded are mainly those from coastal savanna (Adeonipekun 2006).

The pollen zones identified are discussed below.

\section{Zone A (30.0-28.0 m): Transitional Wet Phase}

This is the basal zone of the studied borehole section. It is characterized by a paucity of pollen and spores except for a few spores of Acrostichum aureum, Deltosporites, cf Cyclosorus afar and Nephrolepis; and pollen of Rhizophora spp. and Poaceae as well as fungal spores and hyphae. This zone is associated with Lithofacies types 10 and 9 as described above. Salinity is relatively low (2 ppt compared to the average of $2.2 \mathrm{ppt}$ ). No diatom frustules were recovered. This zone is interpreted as a transitional wet phase due to the dominance of wet paleoclimatic indicator groups over dry. The absence of ferruginous materials within this zone probably lends credence to this interpretation as it may indicate that there was little or no oxidation that is characteristic of exposed littoral sediments during low sea level. From the paleoclimatic inference and its stratigraphic position below a diagnostic dry phase - the Ogolian II, this zone probably represents the latest part of the Inchirian interglacial period. Lack of sufficient organic matter, however, prevented its radiocarbon dating but its age is estimated to be about 35,000 yr BP by extrapolation.

\section{Zone B (28.00-18.00 m): Dry Phase}

This interval is devoid of pollen and spores. The only palynomorphs recovered are fungal elements. From the palynodebris recorded, the lowermost section is dominated by angular to sub-angular brown woods with humic gel and amorphous organic matter (AOM), all forming about $10 \%$ of the ground mass (GM) of the field of view. Within the upper section, the palynodebris recorded abundant AOM (90\%) with humic gel and a few degraded plant materials. These palynodebris are rounded to sub-angular and poorly sorted, occupying $40 \%$ of the field of view. This unit is associated with Lithofacies types $6,7,8$ and 9. Mica flakes and wood materials are present within the lower section while the upper only has ferruginous materials - probably an indication of some oxidation due to exposure. The absence of pollen and spores within this zone is likely due to the dolomite-forming processes indicated in these sediments. Dolomite minerals form as a result of secondary and early diagenetic processes that inhibit the preservation of palynomorphs (Traverse 1988). High dolomites presence in these coastal environments indicates high evaporation during more arid conditions. Salzmann et al. (2002) interpreted high carbonate content as indicating high evaporation in Lake Tilla in northern Nigeria. The palynological barrenness, recovered palynodebris, lithologic parameters and the low salinity, all point to dry paleo-conditions. They are coherent with the Pleistocene Ogolian II regression - an Inter-pluvial phase recognized across Africa (Sowunmi 1981a, 1981b, 1986). This is the first time this 
Pleistocene dry condition is distinctively recognized from any Nigerian basin.

\section{Zone C (18.00-14.00 m): Transitional Dry/Wet Phase}

Though phytoecological groups within this zone are faintly represented, it recorded higher values of WPI with higher proportions of Spores and Rainforest groups relative to the underlying. It is important, however, to report the only recovery of typical savanna pollen Acacia sieberiana within this interval. Even during the previously drier interval, this important pollen was not recovered. The zone is associated with lithofacies types 6 and 5 and its lithology varies from mudstone to indurated sandy clays with some carbonaceous materials. Palynodebris' features are of two types, the Amorphous Organic Matter (AOM) dominated lower section with 40\% Ground Mass (GM), and Brown wood dominated section with $20 \%$ Ground Mass (GM) thereby representing the transition zone from the underlying dry to wet condition. The increase in organic matter, the low salinity and the pollen content suggest that this interval represents a transitional phase from the extreme dry Ogolian II paleoclimate to a wet paleoclimate condition - the African wet paleoclimate maximum (the Pre-Nouaktchottian).

\section{Zone D (14.00-6.00 m): Wet Phase}

This zone is characterized mainly by an abundance of WPI relative to the DPI. It is characterized by abundant Rainforest, Spores, Mangrove and Freshwater phytoecological groups. The dry paleoclimate components such as Poaceae and Open forest vegetation groups, though higher than recorded in the underlying zone, are lower in proportions compared to the wet indicators. In fact, typical rainforest and freshwater tree taxa are restricted to this interval. These tree taxa include Bombax spp., Canthium pollen and Chlorophora excelsa as well as freshwater tree Symphonia globulifera and Uapaca staudtii. Cleome spp., Podococcus barteri, and Eresmopatha are also typical. From the relatively low proportions of the Montane group and the highest spore proportions, these indices indicate a hot humid wet climate. Radiocarbon dating of samples at $12 \mathrm{~m}$ and $6 \mathrm{~m}$ depths of this interval constrains the age of this zone between the basal sample at $15660 \pm 40{ }^{14} \mathrm{C}$ yr BP and its topmost sample at $5495 \pm 25{ }^{14} \mathrm{C}$ yr BP (Table 4). This means that this interval is equivalent to the 12000-5500 YBP wet paleoclimate events of Sowunmi (1986) and 14.8-5.5 cal. Ka BP of DeMenocal et al. (2000) and it would correspond to the African wet paleoclimate maximum - the Pre-Nouaktchottian. Supportive of this inference are the relatively high salinity values and the better presence of diatom taxa compared to previous intervals. However, the sample at $8 \mathrm{~m}$ deep with the highest salinity (5 ppt) is composed only of Coscinodiscus, a brackish water diatom. The disappearance of all freshwater diatoms was likely caused by marine transgression that extended the brackish lagoon water inland. This is also supported by the highest record of Rhizophora spp., high proportion of Poaceae, and Open vegetation groups with a concomitant reduction in the Rainforest group and therefore assigning this sample to a generally wet zone. Palynofacies indices of this interval include the dominance of large-sized greenish-brown woody materials with poor humic gel components but with a progressively high proportion of palynodebris (GM of $20-35 \%$ ). There is no AOM within this interval which was more common in zone $\mathrm{B}$, characterized by the deposition of fine grained dolomites and dry climate. Palynofacies indices of none to poor humic gel contents also indicate low to no oxidation, particularly in the upper section with no record of ferruginous materials at all. The higher sand contents suggest more intense rainfall regimes than in any previous interval. Salzmann et al. (2002) interpreted high quartz contents in Lake Tilla crater in northern Nigeria as indicative of higher input from surrounding slopes during rainfall.

\section{Zone E (4.00-2.00 m): Very Wet and Cold Phase}

This zone has a good representation of all the phytoecological groups with the highest number and diversity of palynomorphs and diatoms. Apart from the highest abundance and diversity of groups, the maximum values of rainforest and mangrove taxa indicate the African maximum marine transgression event reported across Africa (Sowunmi 1986). Surprisingly, however, it has 
a relatively lower value of spores compared to the preceding wet Zone D. Pteridophytes are humid condition indicators and their reduced proportion in this zone indicates that the previous wet zone was more humid. This is supported by the highest proportion of montane Podocarpus milanjianus pollen recovered. That the African maximum transgression event in the Holocene was very wet is a known fact (Sowunmi 1981a, 1981b, 1986, 2004, De Menocal et al. 2000, Salzmann et al. 2000, Ehrmann et al. 2016), but our data suggests that it was a cooler and windy phase as indicated by the peak occurrence of montane pollen - Podocarpus milanjianus. Maley (1991) interpreted the occurrence of montane Olea sp. in Lake Bosumptwi, Ghana, as indicating climatic cooling. Miller \& Gosling (2014) also recovered Olea spp. not only within dry paleoclimatic zone but at the transition to wet paleoclimatic zones (BF3, BF2, and $\mathrm{BF} 1)$ in descending order. They suspected that the Holocene BF1 event was warmer than previous forest events or that other factor might have been responsible for the recovery of Olea. This other factor might have been changes in wind speed, for in this present work, increased wind speed is indicated by the montane Podocarpus milanjianus peak. Adeonipekun et al. (2014) in an aeropalynological study of a site in Ogun State bordering Lagos State in southwest Nigeria, reported the recovery for the first time of bisaccate pollen that is likely to be Podocarpus/Pinus. This recovery, although low in proportion to the traditional pollen of the area, were highest in the month of August when average wind speed was highest in the study area in southwest Nigeria. In this aeropalynological work, the recovery of bisaccates started from the month of June, through July with few grains, peaked in August and nose-dived in September. This confirms the allochthonous source of this pollen which is most likely from the southern extension of the Cameroon mountain ranges into Nigeria as indicated by Keay (1959). Since pollen presence, correlated with increased wind speed, climatic cooling alone could not have been the only factor responsible as reiterated by Miller \& Gosling (2014); rather, the wind speed seems to be a stronger factor. This is also supported by the recovery of the only charred graminoid cuticle found in the whole stratigraphic column and the high abundances of Poaceae pollen at this depth. Radiocarbon dating of the topmost sample of the underlying wet zone $\mathrm{D}$ assigns the sediment a younger age than $5495 \pm 25 \mathrm{yr}$ BP. This, therefore, indicates that this transgression record coincided with the African maximum transgression event - the Nouaktchottian of between 5,500-4,000 yr BP (Sowunmi 1981a, 1981b, 1986).

\section{Zone F: (2.00-0.00 m) Dry Phase}

This is the topmost zone in the studied depth interval and the majority of the botanical evidence suggest dry conditions. The proportion of the DPI is higher than that of the WPI. Of particular significance is the disappearance of most of the forest tree taxa within this zone with the non- recovery of Rainforest and Freshwater groups but little recovery of spores. On the contrary, the Poaceae group had higher proportions supporting drier paleoclimate conditions. The recovered palynodebris is characterized by high particulate amorphous organic matter (PAOM - 80\%), the high recovery of humic gels, and degraded plant materials that are sub-angular to rounded with no woody materials. Lithologically, the sediments are made up of fine-grained sub-rounded sands with lots of carbonaceous detritus and ferruginous materials. These features indicate high oxidation level as confirmed by the reddish brown color of the topmost sediments. It is important to stress that about $0.5 \mathrm{~m}$ of this $2 \mathrm{~m}$ thick sediment is made up of laterite recently used to reclaim the site which would have increased the oxidation indices.

Using the occurrence of ferruginous materials synchronized with pollen and diatom occurrence trends from the base to the top of the studied borehole section, some paleohydrological information can be inferred. The occurrence of ferruginous materials in some saline lakes had been interpreted as indicative of sub-aerial exposure that led to oxidation during a low water level (Valero-Garcés et al. 2004). Its occurrence in the Lagos sequence seems to have a cyclic trend that reflects the bathymetric changes of the depositional site. This trend is synchronous with paleoclimatic changes with high water level being coeval with wet conditions while low water is coeval with dry conditions. The diatom trend of occurrence also shows higher values within the wet high water level phase and low 
values within the low water level in the rich upper section of the borehole. There is no synchronicity between the pollen zones identified and the hydrological cycles. From the occurrence of ferruginous materials, four hydrological cycles were recognized. Each one is characterized by a lower part with an absence of ferruginous materials indicative of no aerial exposure and interpreted as indicative of wetter climatic conditions. The cycle ends with the presence of some ferruginous materials indicative of a lower water level and oxidation. This cycle is repeated four times within the studied borehole although with different magnitude in terms of the hydrological conditions:

1. Cycle 1: $30-28 \mathrm{~m}$ - high water, $28-26 \mathrm{~m}$ - low water;

2. Cycle 2: $26-24 \mathrm{~m}$ - high water, $24-20 \mathrm{~m}$ - low water;

3. Cycle 3: $20-18 \mathrm{~m}$ - high water, $18-10 \mathrm{~m}$ - low water;

4. Cycle 4: $10-4.0 \mathrm{~m}$ - high water, $4.0-0.0 \mathrm{~m}-$ low water.

Cycles 1 and 4 recorded the most extensive high water levels with shorter low water periods while Cycles 2 and 3 recorded shorter high water levels and the most extensive low water levels. These changes are synchronous generally with the wet and dry paleoclimatic changes at a regional scale and could give more information on the paleohydrological changes of the Late Quaternary of West Africa. These paleohydrological reconstructions have helped to shed light on the occurrence of short wet phases within the dry paleoclimatic conditions or short dry phases recorded within generally wet phases. For example, within the very arid Ogolian II, characterized by the deposition of ferruginous materials and interpreted as the last glacial period, two short high water level periods are suggested by the absence of iron-rich deposition. Also, the base of the wettest period of the Early-Middle Holocene - the Pre-Nouaktchottian is also characterized by the deposition of iron-rich materials, which suggests the rapid onset of the African Humid Period. A more detailed biogeochemical analysis of the African coastal deposits is needed to give greater and more concrete insight into the paleohydrological fluctuations and its implications for the Late Quaternary of West Africa. From the recovered palynomorphs, diatoms, and lithological and salinity changes, the environments of the borehole site underwent three major paleoecological changes from approximately $35,000 \mathrm{yr}$ BP. The salinity values of the samples fall within the range generally considered conducive for palynomorph preservation and vary between $0-5$ ppt with an average of 2 ppt. Figure 3 clearly shows that the sediment samples are slightly saline with sample $5(8.0 \mathrm{~m})$ having the highest salinity of $5 \mathrm{ppt}$. This indicates that the location was a marginal inland part of an estuary and never freshwater until the present time. Brackish water salinity ranges between $0.5-17$ ppt while freshwater is less than $0.5 \mathrm{ppt}$ (NOAA 2008). The highest salinity value seems to favor the preservation of palynomorphs as sample 5 has the most diverse palynomorphs. The relatively high salinity seems also to have favored the preservation of fungal elements and reduced the diversity of the mainly freshwater diatom taxa recovered. Guided mainly by the salinity values, the site's paleoecological changes commenced with a brackish water ecosystem such as an inland estuary with a dominance of freshwater spores and some mangroves between intervals of 30-28 m (salinity of $2 \mathrm{ppt}$ ). Following this was a freshwater swamp ecosystem with salinity range of $0-1$ ppt between the intervals of 26-12 $\mathrm{m}$. The relatively lower salinity here, coupled with the dolomitic lithofacies, prevented the recovery of palynomorphs. From this freshwater swamp, the environment changed to a brackish water estuary setting (2-5 ppt) with sample 5 $(8 \mathrm{~m})$ showing maximum salinity. The abundant occurrence of mangrove species and the good preservation of palynomorphs within this interval is characteristic of a brackish water estuary. Today, the location is a freshwater swamp setting with no mangroves, although this is not indicated in the topmost sample due to the laterite and waste water contamination.

\section{CONCLUSION}

The sediments of the coastal area of Lagos, Nigeria in the eastern Benin Basin contain an excellent record of Late Quaternary paleoclimatic changes. The study of palynomorphs, diatom frustules and lithofacies of a $30 \mathrm{~m}$ borehole sedimentary column revealed the extent and magnitude of 
the vegetation and hydrological changes during the last approx. 35,000 yr BP straddling the last glacial and interglacial maxima in West Africa. Cooling and higher wind speeds are most likely associated with the regional African maximum transgression. Four hydrological cycles have been recognized which were more or less synchronous with the wet/dry paleoclimate regional cycles. The Lagos sequence illustrates the potential of coastal swamp deposits as palaeoclimatic and paleoecological archives.

The authors would like to thank Mr. Michael Akinlotan of Horizon Geological Services Ltd., Ibadan, Nigeria for the preparation of the palynological samples. Mr. Keith Richards of KrA Stratigraphic Ltd. UK and Miss Comfort Ogunbanjo of Earthprobe Nigeria Ltd, Lagos assisted immensely in generating the Strataburg distribution charts. Prof. Blas L. Valero Garcés of the Instituto Pirenaico de Ecología-CSIC, Zaragoza, Spain is highly appreciated for his review and comments that improved the manuscript.

\section{REFERENCES}

Adegbie A.T., Schneider R.R., Rohl U. \& Wefer G., 2003. Glacial millenial-scale fluctuations in central African precipitation recorded in terrigenous sediment supply and freshwater signals offshore Cameroon. Palaeogeography, Palaeoclimatology, Palaeoecology, 197, 323-333.

Adekanmbi O.H., Ogundipe O.T. \& Olowokudejo J.D., 2009. Floral Diversity of South-Western Nigeria Coastal Environments. JournalScience Research Development, 11,9-20.

Adeniyi M.O., Ogunsola O.E. Nymphas E.F. \& Oladiran E.O., 2009. Food security measure during uncertain climatic conditions in Nigeria. African Journal of Food, Agriculture, Nutrition and Development, 9, 2, 652-676.

Adeonipekun P.A., 2006. Application of late Tertiary Palynofloral changes as a bio-sequence stratigraphic tool in the western Niger Delta, Nigeria. Department of Botany \& Microbiology, University of Ibadan, Nigeria [Ph.D. thesis, unpublished].

Adeonipekun P.A., Ehinola O.A., Yussuph I.A., Toluhi A. \& Oyelami A., 2012. Bio-sequence stratigraphy of Shagamu Quarry outcrop, Benin basin, southwestern Nigeria. World Applied Sciences Journal, 18, 1, 91-106.

Adeonipekun P.A., Sowunmi M.A. \& Richards K., 2015. A new Late Miocene to Pleistocene palynomorph zonation for the western offshore Niger Delta. Palynology, 41, $1-2,2-16$.

Armitage S.J., Bristow C.S., \& Drake N.A., 2015. West African monsoon dynamics inferred from abrupt fluctuations of Lake Mega - Chad. Proceedings of the National Academy of Sciences of the United States of America, 112, $28,8543-8548$
Billman H.G., 1992. Offshore stratigraphy and paleontology of Dahomey Embayment, West Africa. NAPE Bulletin, $7,21,121-130$.

Coker S.J.L. \& Ejedawe J.E., 1987. Petroleum prospects of the Benin basin, Nigeria. Nigerian Journal of Mining and Geology, 23, 1-2, 27-43.

DeMenocal P., Ortiz J., Guilderson T., Adkins J., Sarnthein M., Baker L. \& Yarusinsky M., 2000. Abrupt onset and termination of the African Humid Period: Rapid climate responses to gradual insolation forcing. Quaternary Science Reviews, 19, 347-361.

Ehrmann W., Schmied G., Seidel M., Krüger S. \& Schulz H., 2016. A distal $140 \mathrm{kyr}$ sediment record of Nile discharge and East African monsoon variability. Climate of the Past, 12, 713-727.

Erdtman G., 1969. Handbook of Palynology. Morphology Taxonomy - Ecology. An Introduction to the Study of Pollen Grains and Spores. Verlag Munksgaard, Copenhagen.

Gasse F., 2000. Hydrological changes in the African tropics since the Last Glacial Maximum. Quaternary Science Reviews, 19, 189-211.

Gosling D., Miller S.M. \& Livingstone D.A., 2013. Atlas of the tropical West African pollen flora. Review of Palaeobotany and Palynology, 199, 1-135.

Hutchinson J. \& Dalziel J.M., 1954. Flora of West Tropical Africa, Vol. 1 Part 1. Crown Agents. London.

Hutchinson J. \& Dalziel J.M., 1958. Flora of West Tropical Africa, Vol. 1 Part 2. Crown Agents. London.

Hutchinson J. \& Dalziel J.M., 1963. Flora of West Tropical Africa, Vol. 2. Crown Agents. London.

Hutchinson J. \& Dalziel J.M., 1968. Flora of West Tropical Africa, Vol. 3 Part 1. Crown Agents. London.

Hutchinson J. \& Dalziel J.M., 1972. Flora of West Tropical Africa, Vol. 3 Part 2. Crown Agents. London.

Jones H.A. \& Hockey R.D., 1964. The Geology of Part of Southwestern Nigeria: Explanation of 1:250,000 Sheets Nos. 59 and 68. Ministry of Mines and Power, Geological Survey of Nigeria Bulletin, 31, Federal Government of Nigeria.

Keay R.W.J., 1959. An outline of Nigerian vegetation. Federal Government of Nigeria, Nigeria.

Lezine M.A., 1989. Late Quaternary Vegetation and climate of the Sahel. Quaternary Research, 2, 317-334.

Longe A., Malomo S. \& Olorunniwo M.A., 1987. Hydrogeology of Lagos metropolis. Journal of African Earth Science, 6, 163-174.

Maley J., 1991. The African rain forest vegetation and palaeoenvironments during late Quaternary. Climate Change, 19, 79-98.

Maley J., 1996. The African rain forest: main characteristics of changes in vegetation and climate from the Upper Cretaceous to the Quaternary. [in:] Alexander I.J., Swaine M.D. \& Watling R. (eds.), Essays on the Ecology of the Guinea-Congo Rain Forest, Proceedings of the Royal Society of Edinburgh, Edinburgh, 104-B, 31-73.

Maley J. \& Brenac, P., 1998. Vegetation dynamics, palaeoenvironments and climatic changes in the forests of western Cameroon during the last 28,000 years B.P. Reviews in Palaeobotany and Palynology, 99, 157-187.

Miller C.S. \& Gosling, W.D., 2014. Quaternary forest associations in lowland tropical West Africa. Quaternary Science Reviews, 84, 7-25. 
NOAA, 2008. Estuary. National Ocean and Atmospheric Administration (Ocean Service Education) US Department of Commerce, [on-line:] http://oceanservice.noaa. gov/education/kits/estuaries/estuaries01_whatis.html [access: 27.02.2017].

Ogbe F.G.A., 1972. Stratigraphy of the strata exposed in the Ewekoro Quarry, western Nigeria. African Geology, 1, 205-322.

Okosun E.A., 1990. A review of the Cretaceous stratigraphy of the Dahomey Embayment, West Africa. Cretaceous Research, 11, 17-27.

Omatsola M.E. \& Adegoke, O.S., 1981.Tectonic evolution and Cretaceous Stratigraphy of the Dahomey Basin. Journal of Mining and Geology, 8, 30-137.

Orijemie E.A. \& Sowunmi M.A., 2014. Humans and the mangrove in southern Nigeria. [in:] Stevens C.J., Nixon S., Murray M.A. \& Fuller D.Q. (eds.), Archaeology of African Plant Use, Publications of the Institute of Archaeology, University College London, 61, Walnut Creek, Left Coast Press, California, 103-112.

Salzmann U., 2000. Are modern savannas degraded forests? - A Holocene pollen record from the Sudanian vegetation zone of NE Nigeria. Vegetation History and Archaeobotany, 9, 1-15.

Salzmann U., Hoelzmann P. \& Morczinek I., 2002. Late Quaternary climate and vegetation of the sudanian zone of Northeast Nigeria. Quaternary Research, 58, 73-83.

Salzmann U. \& Waller M., 1998. The Holocene vegetational history of the Nigerian Sahel based on multiple pollen profiles. Review of Palaeobotany and Palynology, 100, 39-72.

Selley R.C., 1976. Subsurface Environmental Analysis of North Sea Sediments. American Association of Petroleum Geologists Bulletin, 60, 2, 184-195.

Sowunmi M.A., 1973. Pollen of Nigerian Plants. I. Woody species. Grana, 13, 145-186.

Sowunmi M.A., 1981a. Aspects of Late Quaternary vegetational changes in West Africa. Journal of Biogeography, $8,457-474$
Sowunmi M.A., 1981b. Late Quaternary environmental changes in Nigeria. Pollen et Spores, 23, 1, 125-148.

Sowunmi M.A., 1985. The beginning of agriculture in West Africa: Botanical Evidence. Current Anthropology, 26, 1, 127-129.

Sowunmi M.A., 1986. Change of vegetation with time: [in:] Lawson G.W. (ed.), Plant Ecology in West Africa, John Wiley \& Sons, New York, 273-307.

Sowunmi M.A., 1987. Palynological Studies in the Niger Delta. [in:] Alagoa E.J., Anozie F.N. \& Nzewunwa N. (eds.), The Early History of the Niger Delta, Helmat Buske Verlag, Hamburg, UK, 30-31.

Sowunmi M.A., 1995. Pollen of Nigerian Plants. II. Woody species. Grana, 43, 120-141.

Sowunmi M.A., 2004. Aspects of Nigerian coastal vegetation in the Holocene: some recent insights. [in:] Battarbee R.W., Grasse F. \& Stickley C.E. (eds.), Past Climate Variability through Europe and Africa, Springer, Dordrecht, The Netherlands, 199-218.

Tossou M.G., 2002. Recherche palynologique sur la végétation holocène du sud-Bénin (Afrique del'Ouest). Université de Lomé, Faculté des sciences, Togo [Ph.D. thesis, unpublished]

Talbot M.R. \& Delibrias G. 1980. A new late Pleistocene-Holocene water level curve for Lake Bosumptwi, Ghana. Earth Planetary Science Letters, 47, 336-344.

Traverse A., 1988. Production, dispersal and sedimentation of spores/pollen. [in:] Traverse A. (ed.), Paleopalynology, Unwin Hyman, Boston, London, Sydney, Wellington.

Valero-Garcés B.L., González-Sampériz P., Navas A., Machín J., Delgado-Huertas A., Peña-Monné J.L., Sancho-Marcén C., Stevenson T. \& Davis B. 2004. Paleohydrological fluctuations and steppe vegetation at the last glacial maximum in central Ebro valley (NE Spain). Quaternary International, 122, 43-55.

Zabel M., Schneider R.R., Wagner T., Adegbie A.T., de Vries U. \& Kolonic S., 2001. Late Quaternary Climate Changes in Central Africa as Inferred from Terrigenous Input to the Niger Fan. Quaternary Research, 56, 207-217. 